\title{
ÁLCOOL, ALCOOLISMO E ALCOOLISTA: ATITUDES DOS ENFERMEIROS DA ESTRATÉGIA SAÚDE DAFAMÍLIA
}

Maria Elisângela Tavares Bezerra ${ }^{1}$

Noélle de Oliveira Freitas ${ }^{1}$

Fernanda Amendola ${ }^{2}$

Objetivo: avaliar as atitudes dos enfermeiros da Estratégia Saúde da Família-ESF em relação ao álcool, ao alcoolismo e ao alcoolista e correlacionar as atitudes com as práticas, tempo de formação e atuação. Método: Estudo correlacional, observacional, corte transversal realizado nas ESF de oito municipios do Estado de Minas Gerais. Foram aplicados o questionário de avaliação das práticas e a Escala de Atitudes frente ao Álcool, ao Alcoolismo e ao Alcoolista-EAFAAA. Os dados foram analisados por meio da correlação de Spearman. Resultados: 50 enfermeiros participaram do estudo e apresentaram escore total da EAFAAA de 3,1 (DP = 0,4). As correlações entre a EAFAA e o questionário de práticas foram negativas e de fraca intensidade $(-0,23 ; p=0,107)$ e com tempo de formação $(-0,60 ; p=0,679)$ e atuação $(0,01 ; p=0,966)$ foram de fraca intensidade. Conclusão: Os enfermeiros apresentaram tendências a atitudes negativas e não foram evidenciadas correlações entre a EAFAAA com as características dos enfermeiros.

Descritores: Atitude; Atitude do pessoal de saúde. Alcoolismo; Atenção primária à saúde; Enfermagem.

\section{ALCOHOL, ALCOHOLISM AND ALCOHOLISM: ATTITUDES OF NURSES OF THE FAMILY HEALTH STRATEGY}

Objective: To assess the nurses' attitudes from Family Health Strategy-FHS regarding alcohol, alcoholism, and alcoholics and correlate these attitudes with the nurses' practices, time since graduation as a nurse and work at the FHS. Method: Correlational, observational and cross-sectional study performed at the FHS in eight cities in the state of Minas Gerais It was applied the practice assessment questionnaire and the Attitude Scale towards Alcohol, Alcoholism and Alcoholist-EAFAAA. Data were analyzed using Spearman correlation. Results: 50 nurses participated in the study and had a total EAFAAA score of 3.1 (SD = 0.4). The correlations between EAFAA and the practice questionnaire were negative and of low intensity $(-0.23 ; p=0.107)$ and with time of formation (-0.60; $p=0.679)$ and performance $(0.01 ; p=0.966)$ were of low intensity. Conclusion: Nurses showed tendencies towards negatives attitudes and no correlation was found between EAFAAA and nurses' characteristics.

Descriptors: Attitude; Attitude of Health Personnel; Alcoholism; Primary Health Care; Nursing.

\section{ALCOHOL, ALCOHOLISMO Y ALCOHOLISMO: ACTITUDES DE LAS ENFERMERAS DE LA ESTRATEGIA DE SALUD DE LA FAMILIA}

Objetivo: Evaluar las actitudes de los enfermeros de la Estrategia de Salud de la Familia-ESF en relación con el alcohol, alcoholismo y alcoholista y correlacionar las actitudes con las prácticas de los enfermeros, el tiempo de formación y actuación en la ESF. Método: Estudio correlacional, observacional, transversal realizado en la EFS de ocho municipios del estado de Minas Gerais. Fue aplicado el cuestionario de evaluación de la práctica y la Escala de actitud hacia el alcoholismo, el alcoholismo y el alcoholismo-EAFAAA. Los datos se analizaron mediante la correlación de Spearman. Resultados: 50 enfermeros participaron en el estudio y tuvieron un puntaje EAFAAA total de 3.1 (DE = 0.4). Las correlaciones entre EAFAA y el cuestionario de práctica fueron negativas y de baja intensidad $(-0.23 ; p=0.107)$ y con tiempo de formación $(-0.60 ; p=0.679)$ y rendimiento $(0.01 ; p=$ 0.966) fueron de baja intensidad. Conclusión: Los enfermeros presentaron tendencias a actitudes negativas y no se encontró correlación entre EAFAAA y las características de las enfermeras.

Descriptores: Actitud; Actitud del Personal de Salud; Alcoholismo; Atención Primaria de Salud; Enfermería.

IPrograma de Pós-Graduação em Enfermagem da Universidade Univeritas- UNG, Guarulhos, SP. Brasil.

${ }^{2}$ Curso de Graduação e Pós-graduação da Faculdade Israelita de Ciências da Saúde Albert Einstein, São Paulo, SP, Brasil.

Autor correspondente: Noélle de Oliveira Freitas E-mail: nooliveirafreitas@gmail.com

Recebido: 28/10/19 Aceito: 07/06/20 


\section{INTRODUÇÃO}

Nas últimas décadas, o álcool vem sendo uma das substâncias psicoativas mais consumidas no mundo, e o uso e o abuso desta substância têm aumentado drasticamente, constituindo-se em um dos maiores riscos à saúde da população mundial ${ }^{1}$. Segundo dados da Organização Mundial de Saúde (OMS)², o uso do álcool acarreta diversos efeitos nocivos à saúde, prejudicando a qualidade de vida do individuo, além de apresentar problemas de ordem social, de maneira direta e indireta.

Em setembro de 2018, a OMS divulgou o Relatório Global sobre Álcool e Saúde o qual retrata sobre o consumo de álcool no Brasil e no mundo. Foi constatado que, no Brasil, 40\% da população acima de 15 anos consumiu álcool nos últimos 12 meses; em relação ao consumo de álcool per capita foi identificada uma redução em relação ao ano de 2010 de 8,8 litros para 7,8 litros per capita ${ }^{2}$. No tocante aos transtornos relacionados ao uso de álcool, estima-se que 4,2\% dos brasileiros encontram-se nos critérios para abuso ou dependência do álcool, em 2010 a prevalência estimada era de $5,6 \%^{2}$

Diante desse quadro, ressalta-se a importância de que os profissionais que atuam no Sistema Único de Saúde (SUS) envolvam-se com políticas e estratégias para trabalhar com o problema do álcool. Logo, para auxiliar esses profissionais, a OMS apoiou a criação de instrumentos de triagem que servem para identificar o nível de consumo do álcool e outras drogas, auxiliando o profissional de saúde a formular ações interventivas mais adequadas ${ }^{(3)}$. Um desses instrumentos é o Alcohol Use Disorder Identification Test - AUDIT, que foi criado pela OMS para triagem de transtornos relacionados ao álcool ${ }^{4}$

Entretanto, os profissionais da área da saúde encontram dificuldades em lidar com os individuos que apresentam problemas com álcool, e muitas vezes em utilizar esses instrumentos por falta de conhecimento e treinamento ${ }^{5}$. As principais dificuldades dos profissionais surgem devido à carência de formação profissional para atuar com o tema álcool, bem como com as demais substâncias psicoativas o que pode dificultar uma atuação mais efetiva nos problemas relacionados ao álcool e ao alcoolismo ${ }^{\circ}$

Além da falta de conhecimento, as atitudes dos profissionais da área da saúde frente ao tema álcool e alcoolismo é um fator determinante no contexto da assistência em saúde. As atitudes podem ser definidas como a predisposição do indivíduo para agir de maneira igual a uma determinada classe de objetos ou estar sempre pronto para reagir, diante de uma determinada situação ${ }^{7}$. Para a avaliação das atitudes dos enfermeiros em relação ao álcool, ao alcoolismo e ao alcoolista, foi criada a Escala de Atitudes frente ao Álcool, ao Alcoolismo e ao Alcoolista (EAFAAA) ${ }^{8-10}$.

Sendo assim, diante da importância da atuação dos enfermeiros da Estratégia Saúde da Família (ESF) no manejo do tema álcool e alcoolismo na comunidade, o objetivo deste estudo foi avaliar as atitudes dos enfermeiros da ESF em relação ao álcool, ao alcoolismo e ao alcoolista e correlacionar as atitudes com as práticas dos enfermeiros, o tempo de formação e atuação na ESF.

\section{MÉTODO}

\section{Tipo de estudo}

Estudo correlacional, observacional, de corte transversal com abordagem quantitativa.

\section{Participantes da pesquisa}

A população do estudo foi constituida por enfermeiros que atuavam nas equipes de ESF das cidades de Cambuí, Cordislândia, Extrema, Inconfidentes, Itapeva, Pouso Alegre, Monsenhor Paulo e Varginha, do Estado de Minas Gerais. Como critérios de inclusão foram incluidos todos os enfermeiros que atuavam nas equipes de ESF no período da coleta de dados e independentemente do tempo de atuação nas equipes. Foram excluídos os enfermeiros que estavam de férias ou licença médica no período da coleta de dados. Os locais da coleta de dados foram selecionados por conveniência, por ser a região de atuação da pesquisadora principal do estudo.

\section{Local do estudo}

O estudo foi realizado nas equipes de ESF nas cidades de Cambui, Cordislândia, Extrema, Inconfidentes, Itapeva, Pouso Alegre, Monsenhor Paulo e Varginha, do Estado de Minas Gerais.

\section{Coleta dos dados}

A coleta de dados foi realizada pela pesquisadora principal do estudo, no período de julho a agosto de 2017. Para a coleta de dados foi utilizado dois instrumentos: a Escala de Atitudes frente ao Álcool, ao Alcoolismo e ao Alcoolista (EAFAAA) e o questionário de avaliação das práticas dos enfermeiros.

Para avaliação das atitudes dos enfermeiros em relação ao álcool, ao alcoolismo e ao alcoolista foi utilizada a Escala de Atitudes frente ao Álcool, ao Alcoolismo 
e ao Alcoolista (EAFAAA) ${ }^{8.9}$. A EAFAAA foi construida no estudo de Vargas e Luis (2008)(9). No entanto, a versão da EAFAAA utilizada no estudo é composta por 50 itens distribuidos em quatro fatores: fator 1 "O trabaLhar e o relacionar-se com o alcoolista" (21 itens), fator 2 "As atitudes frente ao alcoolista" (9 itens), fator 3 "Atitudes frente ao alcoolismo, etiologia" (1l itens) e fator 4 "As atitudes frente ao uso do álcool" (9 itens)'.

A escala de resposta do EAFAAA é uma escala do tipo Likert, com cinco opções de resposta: 1 - discordo totalmente; 2 - discordo; 3 - indiferente; 4 - concordo; 5 - concordo totalmente ${ }^{8-10}$. Para cálculo do escore total deve-se somar os itens e dividir pelo número de itens do escore total, o mesmo deve ser realizado para cálculo do escore cada fator. A atribuição dos pontos para avaliação da escala corresponde a 1 e 2 pontos para as categorias de respostas desfavoráveis, 3 para pontos no nível intermediário e 4 e 5 pontos para as categorias favoráveis. A EAFAAA tem $75 \%$ dos itens predominantemente negativos e devem ser calculados com valores invertidos. Escores elevados são indicativos de atitudes positivas ${ }^{8-10}$. A EAFAAA foi adaptada e validada para uso no idioma espanhol ${ }^{11}$.

O questionário de avaliação das práticas dos enfermeiros foi desenvolvido pelo grupo de pesquisadores deste estudo os quais atuam em pesquisas na temática da saúde da família. A primeira parte do instrumento contém informações para caracterização dos sujeitos e questões quanto ao conhecimento sobre a quantidade de consumo diário de álcool, recomendado pela OMS para homens e mulheres, quanto à quantidade de álcool considerada de consumo abusivo pelo Ministério da Saúde e se os sujeitos conhecem e já utilizaram o Alcohol Use Disorders Identification Test (AUDIT) ${ }^{(4)}$. Já a segunda parte do questionário tem como objetivo verificar as práticas dos enfermeiros da ESF relacionadas com o álcool, alcoolismo e alcoolista. Foram construidos 18 itens distribuidos em três tópicos. As opções de resposta apresentam-se de acordo com a frequência das práticas com as seguintes opções de resposta: 1 sempre, 2 - regularmente, 3 - às vezes, 4 - raramente e 5 nunca.

\section{Procedimentos de análise de dados}

Os dados do estudo foram inseridos e digitados em duplicidade em uma planilha do Excel ${ }^{\circledR}$ para posterior análise dos dados no programa Statistical $\mathrm{Pa-}$ ckage for the Social Sciences (SPSS) ${ }^{\circledR}$ versão 23.0 para Windows ${ }^{\circledR}$.
Nas variáveis quantitativas, foi testada a distribuição normal pelo teste de Komolgorou-Smirnou. Para a análise de correlação entre os escores da EAFAAA e do questionário de avaliação das práticas dos enfermeiros, foi utilizado o teste paramétrico de correlação não paramétrica de Spearman. Para a comparação entre os escores da EAFAAA versus as variáveis tempo de formação e tempo de atuação como enfermeiro na ESF, foi utilizada a correlação de Spearman. Consideraram-se valores de correlação abaixo de 0,30 como fracas correlações; entre 0,30 e 0,50, moderadas e acima de 0,50 , fortes ${ }^{(12)}$. Os testes foram aplicados para testar as seguintes hipóteses: 1 - as atitudes dos enfermeiros, avaliadas pela EAFAAA, apresentariam correlação positiva e de forte a moderada com a intensidade com as práticas dos enfermeiros em relação ao álcool, ao alcoolismo e ao alcoolista, avaliadas com o questionário de avaliação das práticas dos enfermeiros; 2 - enfermeiros com maior tempo de formação e atuação na ESF apresentariam correlação positiva e de forte a moderada intensidade entre escore total e fatores da EFAAA. Assumiu-se um nível descritivo de 5\% para a significância estatística.

\section{Procedimentos éticos}

O estudo foi aprovado pelo Comitê de Ética em Pesquisa da Universidade Univeritas UNG Guarulhos ( $\mathrm{Pa}$ recer número: 2.149.188; CAAE 66939317.1.0000.5506).

A pesquisadora agendava previamente uma reunião em sala privativa, antes ou após o turno de trabalho dos enfermeiros. Nessa reunião, o enfermeiro foi esclarecido quanto aos objetivos e procedimentos da pesquisa, quanto à liberdade de recusa em participar em qualquer etapa da pesquisa e quanto à garantia de que a informação obtida seria confidencial. Após o aceite em participar da pesquisa, cada enfermeiro assinou o Termo de Consentimento Livre e Esclarecido (TCLE), em duas vias, sendo que uma permaneceu sob a posse da pesquisadora e outra sob a posse do enfermeiro.

\section{RESULTADOS}

A população do estudo foi constituída por todos os enfermeiros que atuavam nas ESF, totalizado 50 enfermeiros. A maioria dos enfermeiros $(96,0 \%)$ era do sexo feminino com a média de idade de 33,8 anos ( $D P=5,7)$. As características sociodemográficas da amostra do estudo estão descritas na Tabela 1 . 
Tabela 1 - Distribuição dos sujeitos do estudo, segundo as variáveis sociodemográficas ( $\mathrm{n}=50$ ). Cambui, Cordislândia, Extrema, Inconfidentes, Itapeva, Pouso Alegre, Monsenhor Paulo, Varginha, MG, Brasil, 2017.

\begin{tabular}{|c|c|c|c|c|c|c|}
\hline Variáveis & $\mathbf{N}$ & $(\%)$ & Mediana & (Intervalo) & Média & $(\mathrm{DP})^{\mathrm{a}}$ \\
\hline \multicolumn{7}{|l|}{ Sexo } \\
\hline Feminino & 46 & $(96,0)$ & & & & \\
\hline Masculino & 4 & $(8,0)$ & & & & \\
\hline \multicolumn{7}{|l|}{ Cidade } \\
\hline Pouso Alegre & 19 & $(38,0)$ & & & & \\
\hline Cambuí & 4 & $(8,0)$ & & & & \\
\hline Itapeva & 3 & $(6,0)$ & & & & \\
\hline Inconfidentes & 3 & $(6,0)$ & & & & \\
\hline Cordislândia & 2 & $(4,0)$ & & & & \\
\hline Tempo de formação (em anos), média, DP* & 50 & $(100)$ & 8,0 & $(<1,0-30,0)$ & 8,6 & $(5,0)$ \\
\hline Pós-graduação & 49 & (98) & & & & \\
\hline Especialista & 26 & (52.0) & & & & \\
\hline Não possui & 16 & (32.0) & & & & \\
\hline Mestrado & 4 & $(8.0)$ & & & & \\
\hline Doutorado & 3 & (6.0) & & & & \\
\hline Tempo de atuação (em anos), média, DP* & 50 & $(100)$ & 5,0 & $(1,0-13,0)$ & 5,1 & $(3,2)$ \\
\hline
\end{tabular}

\section{*Desvio-padrão}

Em relação à avaliação das atitudes, os enfermeiros apresen- $\quad$ com variação de 2,8 a 3,4, entre o escore total e os fatores de taram uma média do escore total da EAFAAA de 3,1 (DP = 0,4) 1 a 4 da EAFAAA (Tabela 2).

Tabela 2 - Estatística descritiva da Escala de Atitudes frente ao Álcool, ao Alcoolismo e ao Alcoolista (EAFAAA) (n=50). Cambuí, Cordislândia, Extrema, Inconfidentes, Itapeva, Pouso Alegre, Monsenhor Paulo, Varginha, MG, Brasil, 2017.

\begin{tabular}{|c|c|c|c|c|c|c|}
\hline Escore total / fatores & Média & DP* & Mediana & $\begin{array}{c}\text { Intervalo } \\
\text { obtido }\end{array}$ & $\begin{array}{l}\text { Intervalo } \\
\text { esperado }\end{array}$ & IC+(95\%) \\
\hline EAFAAA escore total & 3,1 & 0,4 & 3,1 & $2,3-4,0$ & $1-5$ & $3,0-3,5$ \\
\hline EAFAAA Fator lf & 3,4 & 0,5 & 3,4 & $2,1-4,3$ & $1-5$ & $3,3-3,5$ \\
\hline EAFAAA Fator $2 \S$ & 3,3 & 0,4 & 3,4 & $2,3-4,0$ & $1-5$ & $3,2-3,5$ \\
\hline EAFAAA Fator 3 ॥I & 2,8 & 0,6 & 2,7 & $1,8-4,1$ & $1-5$ & $2,6-3,0$ \\
\hline EAFAAA Fator 4 П & 2,8 & 0,5 & 2,8 & $1,3-3,7$ & $1-5$ & $2,7-3,0$ \\
\hline
\end{tabular}

*Desvio-padrão; Intervalo de confiança; O trabalhar e o relacionar-se com o alcoolista; As atitudes frente ao Alcoolista; II Atitudes frente ao alcoolismo, etiologia; As atitudes frente ao uso do álcool 
Para verificar a correlação entre as atitudes e práticas dos enfermeiros, analisou-se a correlação entre a EAFAAA e seus fatores com as práticas dos enfermeiros (Tabela 3).

Tabela 3 - Correlação entre os valores obtidos na aplicação da Escala de Atitudes frente ao Álcool, Alcoolismo e Alcoolista (EAFAAA) e o instrumento para avaliação de práticas dos enfermeiros $(n=50)$. Cambuí, Cordislândia, Extrema, Inconfidentes, Itapeva, Pouso Alegre, Monsenhor Paulo, Varginha, MG, Brasil, 2017.

\begin{tabular}{|c|c|c|}
\hline \multirow{2}{*}{ Instrumentos/Fatores } & \multicolumn{2}{|c|}{ EAFAAA escore total } \\
\cline { 2 - 3 } & $\mathrm{R}$ & $p$-value* \\
\hline EAFAAA Fator $1 \dagger$ & 0,82 & $<0,001$ \\
\hline EAFAAA Fator $2 \neq$ & 0,59 & $<0,001$ \\
\hline EAFAAA Fator 3§ & 0,62 & $<0,001$ \\
\hline EAFAAA Fator 4 $\|$ & 0,12 & 0,400 \\
\hline Práticas dos enfermeiros $\rceil$ & $-0,23$ & 0,107 \\
\hline
\end{tabular}

"p-value - Teste de correlação de Spearman; O trabalhar e o relacionar-se com o alcoolista; As atitudes frente ao alcoolista; SAtitudes frente ao alcoolismo, etiologia; |lAs atitudes frente ao uso do álcool; Questionário de caracterização e avaliação das práticas dos enfermeiros

Observa-se, na Tabela 3, correlação positiva e de forte intensidade entre o escore total da EAFAAA com o fator $1(\mathrm{O}$ trabalhar e o relacionar-se com o alcoolista) $(r=0,82)$, com o fator 2 (As atitudes frente ao alcoolista) $(r=0,59)$ e com o fator 3 (Atitudes frente ao alcoolismo, etiologia) $(r=0,62)$, to- das as correlações estatisticamente significantes $(p<0,001)$. Entretanto, ao correlacionar o escore total da EAFAAA com o questionário de avaliação das práticas dos enfermeiros, foi identificada correlação negativa e de fraca intensidade ( $r=-$ 0,23) e não houve significância estatística.

Tabela 4 - Correlações entre os valores obtidos na aplicação da Escala de Atitudes frente ao Álcool, ao Alcoolismo e ao Alcoolista (EAFAAA) com tempo de formação como enfermeiro e atuação na Estratégia Saúde da Família (ESF) ( $n=50)$. Cambuí, Cordislândia, Extrema, Inconfidentes, Itapeva, Pouso Alegre, Monsenhor Paulo, Varginha, MG, Brasil, 2017.

\begin{tabular}{|c|c|c|c|c|}
\hline \multirow{2}{*}{ Instrumentos/Fatores } & \multicolumn{2}{|c|}{ Formação } & \multicolumn{2}{|c|}{ Atuação na ESF } \\
\hline & $\mathbf{r}$ & $p$-value & r & $p$-value* \\
\hline EAFAAA escore total & -0.60 & 0,679 & 0,01 & 0,966 \\
\hline EAFAAA Fator $1 \dagger$ & -0.21 & 0,153 & $-0,10$ & 0,942 \\
\hline EAFAAA Fator $2 \ddagger$ & 0,03 & 0,854 & 0,18 & 0,201 \\
\hline EAFAAA Fator $\S$ & 0,11 & 0,457 & 0,01 & 0,966 \\
\hline EAFAAA Fator 4\| & 0,12 & 0,424 & 0,01 & 0,959 \\
\hline
\end{tabular}

"p-value - Teste de correlação de Spearman; O trabalhar e o relacionar-se com o alcoolista; As atitudes frente ao alcoolista; Atitudes frente ao alcoolismo, etiologia; |As atitudes frente ao uso do álcool

No que se refere à verificação se enfermeiros com maior tempo de atuação e formação apresentariam maiores atitudes positivas avaliadas pela EAFAAA, observou-se correlação negativa e de forte intensidade entre o escore total da EAFAAA com tempo de formação como enfermeiro, no entanto não houve significância estatística. A correlação entre o escore total da EAFAAA e o tempo de atuação na ESF apresentou-se positiva e de fraca intensidade, não apresentou significância estatística (Tabela 4). 


\section{DISCUSSÃO}

Neste estudo, a maioria dos enfermeiros foi constituída por indivíduos do gênero feminino (96\%) com média de idade de 33,8 anos ( $D P=5,7$ ), indicando que a maioria se encontra entre a população economicamente ativa. Em relação ao gênero, a porcentagem identificada neste estudo corrobora o cenário dos profissionais de enfermagem no Brasil, constituido por $87,2 \%$ de profissionais do gênero feminino, esses dados foram constatados pelo Conselho Federal de Enfermagem (COFEN), em um levantamento em $2011^{13}$.

A hipótese do estudo em relação à correlação das atitudes e às práticas dos enfermeiros em relação ao álcool, ao alcoolismo e ao alcoolista não foi confirmada, sendo identificada correlação negativa entre as atitudes (EAFAAA) e as práticas dos enfermeiros, e esses resultados não foram estatisticamente significativos. Outra hipótese também não confirmada foi a correlação entre as atitudes com tempo de formação como enfermeiro, somente a variável atuação como enfermeiro na ESF apresentou correlação positiva, no entanto de fraca intensidade, e do mesmo modo os resultados não foram estatisticamente significativos.

No tocante à avaliação das atitudes, os resultados deste estudo evidenciaram que os enfermeiros em geral apresentaram atitudes de nivel intermediário para o escore total da EAFAAA, o fator 1 (O trabalhar e o relacionar-se com o alcoolista) e o fator 2 (As atitudes frente ao alcoolista) e atitudes de nível desfavorável no fator 3 (Atitudes frente ao alcoolismo, etiologia) e 4 (As atitudes frente ao uso do álcool). Sendo assim, é possivel afirmar que a maioria dos enfermeiros apresentou tendência a atitudes negativas frente ao álcool, ao alcoolismo e ao alcoolista. Estudo realizado com a EAFAAA identificou valores aproximados ao presente estudo com valor de 3,3 para o Fator 2 (As atitudes frente ao alcoolista $)^{14}$. É importante ressaltar que as atitudes exercem um papel significativo no que tange ao tratamento de pessoas com transtornos relacionados ao uso de álcool?

A versão em espanhol da EAFAAA ${ }^{11}$ foi aplicada com 303 enfermeiros que atuam na área hospitalar assistencial e em serviços de saúde mental na cidade de Bogotá, Colômbia ${ }^{15}$. Neste estudo foi identificado que a maioria dos enfermeiros (56,8\%) apresentou atitudes negativas frente ao álcool, ao alcoolismo e ao alcoolista com média da EAFAAA de 3,0 para mulheres e 3,1 para homens ${ }^{(15)}$ corroborando com os resultados do presente estudo no qual os enfermeiros em seu total apresentaram média de escore total da EAFAAA de 3,1 $(D P=0,4)$. Ao correlacionar o escore da EAFAAA com as características dos enfermeiros, a variável tempo de graduação dos enfermeiros colombianos teve correlação positiva e de forte intensidade com os escores da EAFAAA ( $r=0,80 ; p<0,0001$ ), ou seja, enfermeiros com maior tempo de formação apresentam atitudes mais positivas ${ }^{(15)}$, no entanto tal correlação não foi identificada neste estudo.

Um estudo desenvolvido na Inglaterra em 2012 avaliou as atitudes em relação aos pacientes com problemas com álcool de profissionais de três hospitais. Participaram do estudo 204 profissionais sendo 145 enfermeiros $(71,1 \%)$ os quais responderam o instrumento Short Alcohol and Alcohol Problems Perception Questionnaire (SAAPPQ)(15). Os resultados do estudo apontaram que os profissionais em geral apresentaram uma atitude um pouco mais positiva em relação aos pacientes com problemas com álcool. O fato do profissional trabalhar no departamento de dependência e psiquiatria do hospital foi um fator preditor das atitudes em relação aos pacientes com problemas com álcool, indicando que fato de serem treinados e trabalharem em um local especializado teve uma influência positiva nas atitudes da equipe ${ }^{(15)}$. Os resultados deste estudo em geral se diferem aos do estudo realizado na Inglater$\mathrm{ra}^{(15)}$, pois as atitudes dos enfermeiros em relação ao álcool avaliadas por meio da EAFAAA apresentaram tendência negativa, embora a avaliação das atitudes tenha sido realizada por meio de escalas distintas o que dificulta uma comparação mais legítima entre os resultados dos estudos.

Um estudo qualitativo realizado na Finlândia com enfermeiros que participaram de um treinamento online sobre o atendimento de pacientes intoxicados por álcool atendidos em serviços de emergência identificou três categorias nas análises dos discursos: a falta de compromisso dos enfermeiros com o atendimento intoxicado ao paciente, o compromisso dos enfermeiros com o atendimento intoxicado ao paciente e as habilidades dos enfermeiros nos métodos de intervenção ${ }^{17}$. Os enfermeiros consideraram que o aumento da conscientização por meio de treinamentos sobre o atendimento aos pacientes com intoxicação por álcool pode contribuir para o desenvolvimento de atitudes mais positivas e habilidades dos enfermeiros no atendimento destes pacientes nos serviços de emergência ${ }^{17}$. 
No Brasil, pesquisa evidenciou que os enfermeiros de atenção primária são favoráveis ao tratamento do alcoolismo, sendo o paciente percebido como doença ${ }^{(18)}$. Os enfermeiros que atuam na atenção primária apresentaram atitudes negativas ao trabalhar com esta clientela quando comparados aos enfermeiros que trabalham em unidades hospitalares ${ }^{18}$.

No Brasil, uma pesquisa evidenciou que os enfermeiros que atuam na atenção primária apresentaram atitudes negativas em relação a pessoas em tratamento do alcoolismo quando comparados aos enfermeiros que atuam em unidades hospitalares ${ }^{18}$. Os enfermeiros que atuam na atenção primária, inclusive aqueles que atuam na Estratégia Saúde da Família, ainda têm dificuldade em distinguir as ações voltadas a promoção da saúde das ações de prevenção de doenças ${ }^{19}$. No tocante ao tratamento do alcoolismo, os enfermeiros de atenção primária são favoráveis ao tratamento sendo o paciente percebido como doença ${ }^{18}$, ao invés de terem como foco a promoção da saúde no contexto do alcoolismo.

Um estudo que avaliou os artigos produzidos pela mídia impressa no Brasil nos anos de 2005 a 2010 com o objetivo de caracterizar as representações sociais sobre os efeitos da bebida alcoólica concluiu que os resultados podem contribuir para os enfermeiros conhecerem melhor sobre o fenômeno da bebida alcoólica e do alcoolismo e, por meio da educação em saúde promoverem a formação e capacitação de novos profissionais de saúde visando a redução a redução dos problemas com álcool no Brasil ${ }^{20}$.

Um estudo realizado em um hospital geral avaliou as atitudes dos enfermeiros em relação ao alcoolismo e constatou que os enfermeiros reconhecem que o alcoolismo é uma doença, entretanto apresentam atitudes negativas, ao afirmarem que preferem não trabalhar com esta clientela, evidenciando um forte valor moralista ${ }^{21}$. Outro estudo realizado com enfermeiros de um hospital filantrópico sobre o cuidado a indivíduos alcoolistas também constatou que a maioria dos enfermeiros reconhece que o alcoolismo é uma doença crônica que envolve fatores biopsicossociais e que necessita de tratamento, no entanto o cuidado de enfermagem ainda está permeado por uma estigmatização dos alcoolistas ${ }^{22}$.

Em relação ao conhecimento sobre questões relacionadas ao álcool e ao alcoolismo, os enfermeiros apresentam dificuldade em reconhecer os problemas relacionados ao uso do álcool. O preparo dos enfermeiros e o conhecimento relacionam-se diretamente às atitudes positivas frente ao alcoolista. Os resultados deste estudo identificaram que a maioria dos enfermeiros não tem conhecimento sobre as principais diretrizes relacionadas ao consumo de álcool. A capacitação e o preparo dos enfermeiros contribuem positivamente com a mudança das atitudes desses profissionais, diminuindo as atitudes de moralização frente ao uso do álcool ${ }^{14}$. Além disso, o fato do profissional ser treinado e trabalhar em um local especializado pode melhorar positivamente as atitudes da equipe em relação ao paciente com problemas com o uso do álcool ${ }^{16}$.

\section{Limitações do Estudo}

O estudo apresenta uma limitação que merece ser comentada no tocante a escassa produção científica na área do estudo. A escassa produção de estudos nesta área sobre as atitudes dos enfermeiros em relação ao álcool, ao alcoolismo e ao alcoolista dificultou uma discussão mais abrangente sobre a temática.

\section{Contribuições para a Prática}

O estudo possui relevância para a prática, pois fornece subsidios para o compreendimento das atitudes dos enfermeiros de ESF frente ao álcool, ao alcoolismo e ao alcoolista. Além disso, os achados descritos fornecem subsídios para uma reflexão sobre a importância da mudança das atitudes dos profissionais diante da problemática investigada.

\section{CONCLUSÃO}

O estudo permitiu identificar que, no geral, estes profissionais apresentaram tendências a atitudes negativas frente ao álcool, ao alcoolismo e ao alcoolista e, além disso, o tempo de formação como enfermeiro e a atuação na ESF não apresentaram correlação com as atitudes. Novos estudos devem ser desenvolvidos a nível de ESF para permitir a identificação e contribuir para o aperfeiçoamento da assistência ao usuário de álcool, compreender sobre as questões relacionadas ao álcool, ao alcoolismo e ao alcoolista, além de permitir a reflexão sobre a mudança das atitudes dos profissionais.

Contribuições dos Autores: Todos os autores contribuíram em todas as etapas do estudo. 


\section{REFERÊNCIAS}

1. World Health Organization. 2009 [acesso em 07 set 2016]. Disponivel em http://www.who.int/substance_abuse/activities/public_health_alcohol/en/

2. World Health Organization. Global status report on alcohol and health 2018. Geneva: World Health Organization; 2018 [acesso em 23 set 2018]. Disponivel em: http://www.who.int/substance_abuse/publications/global_alcohol_report/en/

3. Vargas D. Rastreamento de individuos com dependência alcoólica em serviços de atenção básica a saúde. IN: 10 Seminário Internacional da Rede de Pesquisa sobre Drogas.2007 out.4-5; Brasília, DF, BR. Anais. Brasilia: Secretaria Nacional Antidrogas; 2007.

4. Saunders JB, de la Fuente JR, Grant M. Development of the Alcohol Use Disorders Identification Test (AUDIT): WHO Collaborative Project on Early Detection of Persons with Harmful Alcohol Consumption--II. Addiction. 1993;88(6):791-804.

5. Crothers CE, Dorrian J. Determinants of Nurses' Attitudes toward the Care of Patients with Alcohol Problems. ISRN Nurs. 2011; 821514. doi: http://dx.doi.org/10.5402/2011/821514

6. Vargas D, Duarte FAB. Enfermeiros dos centros de atenção psicossocial em álcool e drogas (caps ad): a formação e a busca pelo conhecimento especifico da área. Texto contexto - enferm. 2011; 20 (1): 119-126. doi: http://dx.doi.org/10.1590/ s0104-07072011000100014

7. Rosenberg MJ, Horland CL. Atitude, organization and change: Na analysis of consistency among atitude componentes. New Haven: Yale University Press;1960.

8. Vargas D, Luis MAV. Construção e validação de uma escala de atitudes frente ao álcool, ao alcoolismo e ao alcoolista. Rev. Latino-Am. Enfermagem. 2008; 16 (5): 895-902. doi: http://dx.doi.org/10.1590/S0104-11692008000500016

9. Merces, NP. Atitudes dos estudantes de psicologia acerca do álcool, do alcoolismo e do alcoolista. 2013. [dissertação]. São Paulo: Escola de Enfermagem, Universidade de São Paulo, Ribeirão Preto.

10. Vargas D. Validação de construto da escala de atitudes frente ao álcool, ao alcoolismo e a pessoas com transtornos relacionados ao uso do álcool. Rev. Psiquiatr. Clín. 2014; 41 (4): 106-111. doi: http://dx.doi.org/10.1590/0101-60830000000021 11. Ramirez EGL, Vargas D. Escala de atitudes frente ao álcool versão em espanhol: evidências de validade e confiabilidade. Rev. Latino-Am. Enfermagem. 2017; 25: e2918. https://doi.org/10.1590/1518-8345.1721.2918

12. Ajzen J, Fishben M. Overview. Understanding attitudes and predicting social behavior. New Jersey: Prentice Hall, 1998.

13. Barreto IS, Krempel MC, Humerez DC. O Cofen e a Enfermagem na América Latina. Enfermagem em Foco 2011; 2(4):251254.

14. Caixeta LMM, Pedrosa LAK, Hass VJ. Análise das atitudes de profissionais da Atenção Primária a Saúde frente a pessoas com transtornos relacionados ao uso de álcool. SMAD, Rev. Eletrônica Saúde Mental Álcool Drog. 2016; 12 (2): 84-91. https://doi.org/110.11606/issn.1806-6976.v12i2p84-91

15. Ramirez EGL, Vargas D, Luis MV. Atitudes frente ao álcool, ao alcoolismo e à pessoa com transtornos relacionados ao uso de álcool em enfermeiros colombianos. Cogitare enferm. 24: e58795, 2019. https://doi.org/110.5380/ce.v24i0.58795

16. Iqbal N, McCambridge O, Edgar L, Young C, Shorter GW. Health-care professionals' attitudes across different hospital departments regarding alcohol-related presentations. Drug and Alcohol Review (September 2015), 34, 487-494. https://doi. org/10.11ll/dar.12243

17. Hakala T, Kylmä J, Mäkelä KL, Noppari E, Koivunen M.Caring for alcohol-intoxicated patients in an emergency department from the nurses' point of view - focus on attitudes and skills. Scand J Caring Sci. 2020 Feb 9 [Epub ahead of print]. https://doi.org/10.1111/scs.12825

18.Vargas D, Labate RC. Trabalhar com pacientes alcoolistas: satisfação de enfermeiros de hospital geral. Rev Gaúch Enferm. 2005;26(2);252-260.

19. Moll MF, Boff NN, Silva PS, Siqueira TV, Ventura CAA. O enfermeiro na saúde da família e a promoção de saúde e prevenção de doenças. Enferm. Foco 2019; 10 (3): 134-140. https://doi.org/10.21675/2357-707X.2019.v10.n3

20. Conceição VM, Silva SED, Araujo JS, Santana ME, Vasconcelos EV. As representações sociais da bebida alcoólica e suas consequências na sociedade expressas pela midia impressa. Enfermagem em Foco. 2012; 3(1):42-45.

21. Vargas D. Atitudes de enfermeiros de hospital geral frente ao paciente alcoolista. [dissertação de mestrado]. Ribeirão Preto (SP): Escola de Enfermagem/USP; 2001

22. Barbosa, LN, Mangueira OS, Albuquerque GJ, Guimaraes JF. Cuidado de Enfermagem a pacientes alcoolistas: percepções da equipe de enfermagem. Rev. Bras. Pesq. Saude 2013; 15 (2): 88-93. 